\title{
SI on computational methods for network optimization problems
}

\author{
Bernard Fortz • Luis Gouveia
}

Published online: 6 November 2013

(C) Springer-Verlag Berlin Heidelberg and EURO - The Association of European Operational Research Societies 2013

This special double issue of the EURO Journal on Computational Optimization focuses on computational methods for network optimization problems. Network optimization is a key area of operational research. Significant research activities are produced in this topic, as attested by the success of conferences organized by the EURO working group "European Network Optimization Group" (ENOG).

The first volume of this special double issue is organized as follows.

The paper by Bley, Ljubić and Maurer presents Lagrangian decomposition methods for problems arising in two-level optical networks. These problems are very sensitive for network operators today as the tendency is to upgrade networks to bring fiber optic cables closer to the clients.

Next, Claßen, Koster and Schmeink develop improvements to speed up a column generation scheme for robust wireless network planning. In a world of mobile devices, the planning of wireless networks is of crucial importance. The paper describes useful computational tools to achieve an effective robust planning.

Finally, Groiez, Desaulniers, Hadjar and Marcotte investigate how to solve a multicommodity network flow formulation of the multiple depot vehicle scheduling problem, using a branch-and-cut algorithm that incorporates an efficient implementation of a separation procedures for odd-cycle and odd-set inequalities.

We would like to thank the authors for having submitted top quality papers and the referees for their excellent reviews.

\footnotetext{
B. Fortz $(\varangle)$

Département d'informatique, Université Libre de Bruxelles, Brussels, Belgium

e-mail: bernard.fortz@ulb.ac.be

L. Gouveia

Centro de Investigação Operacional, Faculdade de Ciências,

Universidade de Lisboa, Lisbon, Portugal

e-mail: legouveia@fc.ul.pt
} 